\title{
Etude expérimentale de l'influence de Tubifex tubifex Müll. (Tubi- ficidae, Oligochaeta) sur le relargage du phosphore par le sédiment
}

\author{
C. Guérin ${ }^{1}$ \\ L. Labrouel
}

Mots clés : Tubifex, sédiment, phosphore, relargage.

L'jnfluence de Tubificidae du genre Tubifex sur le relargage des différentes formes du phosphore (particulaire, organique dissous et orthophosphates) a été étudiée au laboratoire, en conditions contrôlées. Lorsque le milieu est oxygéné, les vers entraînent une augmentation du phosphore particulaire dans la colonne d'eau mais aux dépens des orthophosphates, de sorte que le relargage de phosphore total n'est guère supérieur à celui du témoin. En milieu appauvri en oxygène, la différence est plus nette mais elle est surtout due au phosphore particulaire plus abondant. Ce phosphore particulaire semble provenir d'une agitation plus importante car la défécation est réduite par le déficit en oxygene. Le transfert apparent orthophosphates-phosphore particulaire pose le problème de la biodisponibilité de ce dernier qui conditionne l'action positive ou négative des Tubifex sur la nutrition algale.

A laboratory study of the effect of Tubifex tubifex Müll. (Tubificidae, Oligochaeta) on the release of phosphorus through the sediment

Keywords : Tubifex, sediment, phosphorus, release.

The effect of Tubificidae of the genus Tubifex on the release of different forms of phosphorus (particulate, dissolved organic and orthophosphates) has been studied in the laboratory under controlled conditions. When the environment is oxygenated, the worms produce an increase in particulate phosphorus in the water column, but at the expense of orthophosphates so that the amount of total phosphorus is scarcely higher than that of a control. When the environment has a low oxygen content, the difference is more marked, chiefly due to the greater abundance of particulate phosphorus. This particulate phosphorus appears to arise from more essential stirring because defaecation is reduced by the oxygen deficit. This transfer of orthophosphates to particulate phosphates raises the problem of the biological availability of the latter that condition the positive or negative action of Tubifex on algal nutrition.

\section{Introduction}

Les Tubificidae du genre Tubifex sont des Oligochètes aquatiques inféodés aux sédiments vaseux de la plupart des écosystèmes d'eau douce. Ils contribuent, avec d'autres organismes benthiques tels que les Chironomidae, les Amphipodes, les Unionacae et les Pisidiidae, au mélange du sédiment dans lequel ils vivent (Mc Call et al. 1982). Leur comportement alimentaire les conduit à ingérer, lors de leurs déplacements, les particules sédimentaires yui sont sur leur passage, de façon à prélever leur

1. Laboratoire d'Hydrobiologie, U.R.A. 695 C.N.R.S., Université Paul Sabatier, 118, route de Narbonne, 31062 Toulouse Cedex, France. nourriture (bactéries, algues, détritus selon les espèces) puis à les déféquer en un lieu différent de celui de la prise alimentaire. L'activité mécanique des Tubificidae (fouissage, déplacement), ainsi que leur activité physiologique (défécation, excrétion), tendent à modifier les caractéristiques physicochimiques du milieu et à influencer la distribution de substances dissoutes (Petr 1977, Mc Call \& Fisher 1980, Krantzberg 1985). L'influence des Oligochètes, plus particulièrement sur le phosphore, a fait l'objet de plusieurs études. Les avis concernant l'importance du rôle des vers sur le cycle du phosphore divergent. Parmi les auteurs qui ont mis en évidence une action des Oligochètes, certains mettent en cause l'excrétion (Fukuhara \& Sakamoto 1987), d'autres la circulation d'eau provoquée par le déplacement et le fouissage des vers (Wisniewski \& Planter 1985). 
Le but de notre étude a été d'évaluer si les Tubificidae intervenaient ou non sur le relargage du phosphore par le sédiment en milieu expérimental et, si oui, de quelle façon et dans quelles proportions leur influence était-elle significative.

\section{Matériel et méthodes}

L'expérience de relargage du phosphore par le sédiment a été réalisée dans des aquariums étroits en verre (fig. 1), ce qui permet l'utilisation d'une petite quantité de sédiment et d'Oligochètes (reproductibilité, économie du matériel vivant) tout en offrant la possibilité de travailler sur des densités de population élevées.

Les mesures d'excrétion ont eu lieu dans des piluliers de $120 \mathrm{ml}$ munis d'un filet (vide de maille de $25 \mu \mathrm{m}$ ) où ont été déposés les Oligochètes (fig. 2). Ce dispositif permet une séparation rapide et fine du matériel dissous excrété et du matériel solide (fécès) déféqué par les vers.

Les Oligochètes du genre Tubifex (Tubificidae) ont été récoltés un mois environ avant le début de l'expérience et maintenus à $22^{\circ} \mathrm{C}$ au laboratoire dans un aquarium.

Le sédiment provient d'un petit lac de gravière hypereutrophe et riche en phosphore, situé à Layrac, à environ $30 \mathrm{~km}$ au nord de Toulouse. Il a été récolté quelques jours avant le début de l'expérience à l'aide d'une drague Ekman. Au laboratoire, il est réparti dans les aquariums, à raison de $350 \mathrm{~cm}^{3} \mathrm{de}$ sédiment par aquarium. Ces derniers ont été placés deux heures à l'étuve à $70^{\circ} \mathrm{C}$ de façon à éliminer la faune et les cocons de vers qui s'y trouvent. Les aquariums sont ensuite disposés à l'obscurité à une température de $20^{\circ} \mathrm{C}$. L'expérience n'a commencé que cinq jours plus tard, lorsque toutes les particules ont sédimenté et que le milieu a atteint la température ambiante.

28 aquariums ont été préparés en vue de l'expérience. L'eau qui se trouvait à la surface des

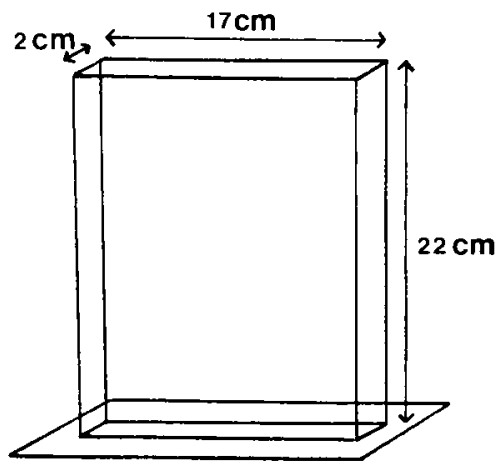

Fig. 1. Aquarium étroit en verre.

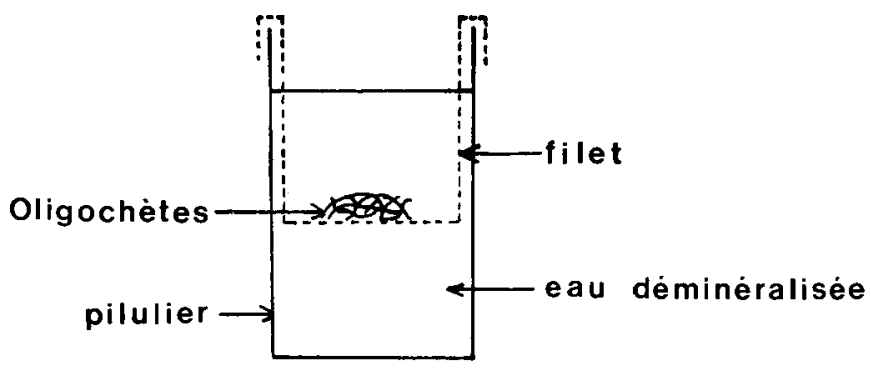

Fig. 2. Dispositif utilisé pour l'excrétion des Tubificidae. 
sédiments a été siphonée et remplacée par $400 \mathrm{ml}$ d'eau déminéralisée. Le milieu est aéré grâce à un léger bullage. 50 Tubifex ont alors été ajoutés dans 20 des 28 aquariums, ce qui correspond à une densité de population égale à environ 14300 individus $/ \mathrm{m}^{2}$. Les 8 aquariums sans vers servent de témoin. L'ensemble des aquariums a été placé à l'obscurité et maintenu à environ $20^{\circ} \mathrm{C}$ pendant les 12 jours que dure l'expérience. Si besoin, le niveau d'eau surnageante a été réajusté à $400 \mathrm{ml}$.

Le sixième jour, $300 \mathrm{ml}$ d'eau superficielle ont été prélevés dans tous les aquariums, sauf dans 4 contenant des Oligochètes. Le sédiment des 8 aquariums avec vers est tamisé de façon à recueillir ces derniers. Les Tubificidae sont alors précautionneusement lavés cinq fois dans de l'eau déminéralisée. Chaque lot de 50 vers est déposé à l'intérieur d'un filet fixé sur un pilulier en verre contenant $100 \mathrm{ml}$ d'eau déminéralisée. Après 5 heures, les filets sont sort is des piluliers. Les Oligochètes sont recueillis et placés à l'étuve à $80^{\circ} \mathrm{C}$ pendant 24 heures, pour en déterminer le poids sec. Les fécès piégées par les filets sont récupérées sur filtre (nuclépore $0,4 \mu \mathrm{m}$ ) en vue des dosages. L'eau déminéralisèe dans laquelle a eu lieu l'excrétion par les vers est conservée pour les dosages.

Les 4 aquariums, dans lesquels l'eau superficielle n'a pas été prélevée, continuent à être aérés. Les autres, après que leur niveau ait été réajusté à $\mathbf{4 0 0} \mathrm{ml}$ avec de l'eau déminéralisée, sont recouverts d'une couche d'huile de vaseline, afin d'empêcher les échanges avec l'air.

Au douzième jour, en fin d'expérience, $300 \mathrm{ml}$ d'eau surnageante sont prélevés dans les aquariums restant. Les vers des aquariums aérés et des aquariums recouverts d'huile sont récupérés et placés dans les pilluliers pendant 5 heures pour estimer l'excrétion et la défécation.

Le phosphore total, les orthophosphates et les matières en suspension sont mesurés sur chaque échantillon d'eau superficielle.

Le phosphore total dissous et les orthophosphates sont mesurés sur les $100 \mathrm{ml}$ d'eau diméralisée dans laquelle a eu lieu l'excrétion.

Le phosphore total est dosé sur les fécès ainsi que sur les matières en suspension.
Les orthophosphates sont dosés par la méthode colorimétrique au vert de malachite/molybdate d'ammonium exposée par Motomizu et al. (1983). Le phosphore total et le phosphore total dissous nécessitent une minéralisation à l'autoclave avant dosage par cette même méthode. Le phosphore organique dissous est obtenu par différence entre le phosphore total dissous et les orthophosphates.

\section{Résultats}

\subsection{Relargage du phosphore dans la colonne d'eau en milieu oxygéné}

\section{Echanges sédiment-eau}

La quantité de phosphore total relarguée par le sédiment, dans un milieu dont le pourcentage de saturation en oxygène est de $120 \% \pm 2$ (Tableau I), est légèrement plus importante dans un sédiment qui contient des Oligochètes que dans un sédiment qui n’en contient pas : 3,82 mg de phosphore libérés par $\mathrm{m}^{2}$ par jour, contre $3,49 \mathrm{mg}$ (fig. 3, Tableau II A). On observe $2,52 \mathrm{mg}$ de phosphore liberés par $\mathrm{m}^{2}$ par jour, sous forme d'orthophosphates, par un sédiment sans Tubificidae, contre $0,94 \mathrm{mg}$ dans un sédiment avec Tubificidae, $0,8 \mathrm{mg}$ sous forme organique dissous contre $1,29 \mathrm{mg}$, et, entin, $0,17 \mathrm{mg}$ sous forme particulaire contre $1,59 \mathrm{mg}$ (fig. 3 , Tableau II A).

La présence des Oligochètes est donc responsable, à elle seule, du relargage par le sédiment, de $1,42 \mathrm{mg}$ de phosphore par $\mathrm{m}^{2}$ par jour, sous forme particulaire, de $0,49 \mathrm{mg}$ sous forme organique dissous, et de la fixation par le sédiment de $1,58 \mathrm{mg}$ de phosphore sous forme d'orthophosphates. Le bilan donne un relargage supplémentaire de phosphore total par les sédiments de $0,33 \mathrm{mg}$ par $\mathrm{m}^{2}$ par jour, dû à la présence des Oligochètes dans le sédiment (fig. 4), qui n'est pas significatif et ne reprèsente que $8,6 \%$ du phosphore total libéré par le sédiment.

Par contre, les proportions des différentes formes de phosphore relarguées varient significativement (sauf pour le phosphore organique dissous) selon que le sédiment contient des vers ou pas. Ainsi, dans un milieu sans Oligochètes, $95 \%$ du phosphore libéré est sous forme dissous contre $59 \%$ en présence de vers $(f i g .5 \mathrm{~A}$ ). Inversement, $42 \%$ du phosphore relargué par le sédiment contenant des Tubifex est sous forme particulaire, contre seulement $5 \%$ dans un milieu sans Oligochètes (fig. 5 A). 
Tableau I. Pourcentage de saturation en oxygène dans les différents miliẹx.

\begin{tabular}{llc}
\hline & Milieu aéré & Milieu non aéré \\
\hline Témoin sans vers & $120,00 \pm 2$ & $45,00 \pm 5.9$ \\
Milieu avec vers & $122,00 \pm 2$ & $46,25 \pm 1,9$ \\
\hline
\end{tabular}

Tableau II A. Taux de relargage (en $\mathrm{mg} / \mathrm{m}^{2} / \mathrm{j}$ ) des différentes formes de phosphore par le sédiment, en rnilieu aéré, en présence ou non d'Oligochètes.

\begin{tabular}{lcc}
\hline & Témoin sans vers & Milieu avec vers \\
\hline P total & $\mathbf{3 , 4 9} \pm 1,85$ & $\mathbf{3 , 8 2} \pm \mathbf{1 , 7 1}$ \\
P particulaire & $\mathbf{0 , 1 7} \pm \mathbf{0 , 0 0 9}$ & $\mathbf{1 , 5 9} \pm 0,92$ \\
P total dissous & $\mathbf{3 , 3 2} \pm 1,76$ & $2,23 \pm 0,79$ \\
Orthophosphates & $\mathbf{2 , 5 2 \pm 1 , 7}$ & $0,94 \pm 0,36$ \\
P organique dissous & $\mathbf{0 , 8} \pm \mathbf{0 , 2 4}$ & $1,29 \pm 0,43$ \\
\hline
\end{tabular}

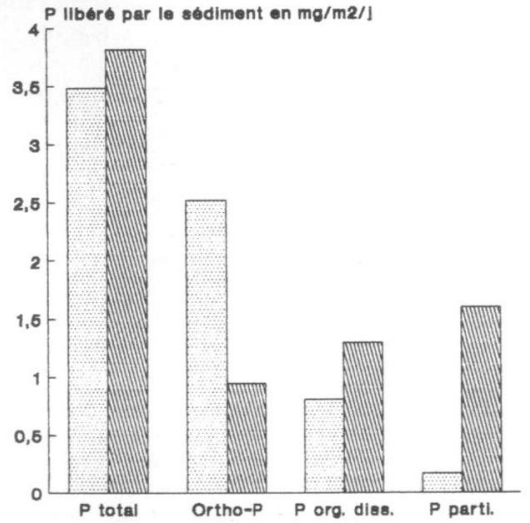

Milleu sans vers MIIIIII Milieu avec vera

Fig. 3. Influence des Tubificidae sur le relargage des différentes formes de phosphore, dans un milieu oxygéné.
Tableau II B. Taux de relargage (en $\mathrm{mg} / \mathrm{m}^{2} / \mathrm{j}$ ) des différentes formes de phosphore par le sédiment, en miljeu non aéré, en présence ou non d'Oligochètes.

\begin{tabular}{lcc}
\hline & Témoin sans vers & Milieu avec vers \\
\hline P total & $5,48 \pm 2,32$ & $8,92 \pm 3,67$ \\
P particulaire & $3,63 \pm 2,7$ & $7,12 \pm 3,92$ \\
P total dissous & $1,85 \pm 0,38$ & $1,8 \pm 0,6$ \\
Orthophosphates & $1,28 \pm 0,19$ & $0,93 \pm 0,3$ \\
P organique dissous & $0,57 \pm 0,29$ & $0,86 \pm 0,31$ \\
\hline
\end{tabular}

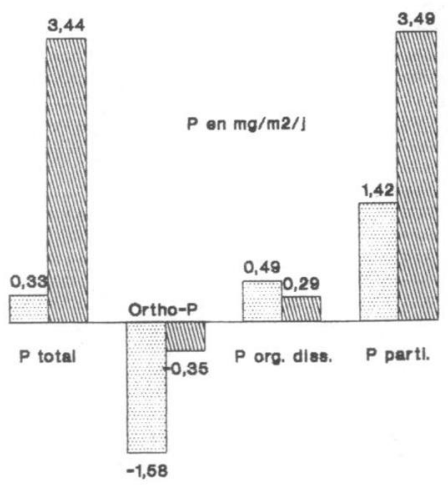

Fig. 4. Taux de phosphore, en $\mathrm{mg} / \mathrm{m}^{2} / \mathrm{j}$, relargué ou retenu par le sédiment, grâce aux Tubificidae. 


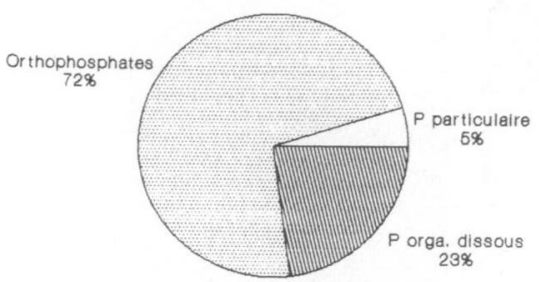

Milieu sans Oligochètes

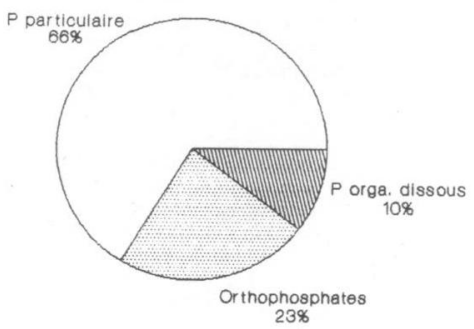

Milieu sans Oligochètes
A

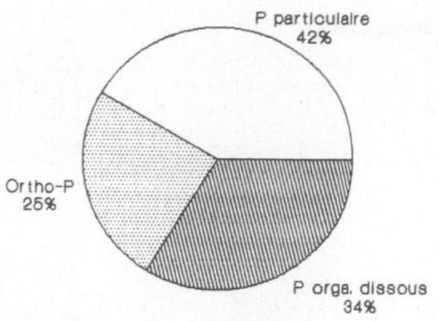

Milieu avec Oligochètes

B

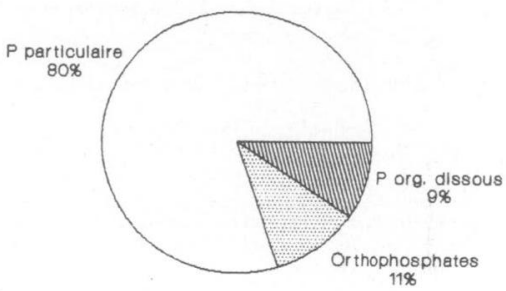

Milieu avec Oligochètes

Fig. 5. Répartition des différentes formes de phosphore relarguées par $\mathrm{m}^{2}$ et par jour par le sédiment. (A : milieu oxygéné ; B : milieu non oxygéné).

On constate d'ailleurs parallèlement que les matières en suspension atteignent $50 \mathrm{mg} / \mathrm{l}$ dans un milieu contenant des vers, contre seulement $10 \mathrm{mg} / \mathrm{l}$ dans un milieu qui n'en contient pas (fig. 6, Tableau III).

\section{Excrétion - défécation}

Le taux de défécation, en milièu bien aéré, est de $4,9 \mathrm{mg}$ de sédiment sec par $\mathrm{m}^{2}$ par jour (fig. 7 , Tableau IV).
La quantité de phosphore total libéré directement grâce à l'excrétion et la défécation par des vers, ayant séjourné six jours dans un milieu bien aéré, est de $20,42 \mathrm{mg}$ par $\mathrm{m}^{2}$ par jour (fig. 8, Tableau $\mathrm{V}$ ). La répartition de ce phosphore est la suivante: 2,9 mg par $\mathrm{m}^{2}$ par jour (soit $14 \%$ ) de phosphore, sous forme d'orthophosphates, et $1,64 \mathrm{mg}$ par $\mathrm{m}^{2}$ par jour (soit $8 \%$ ) de phosphore, sous forme particulaire (fig. $8 \& 9$, Tableau V). 


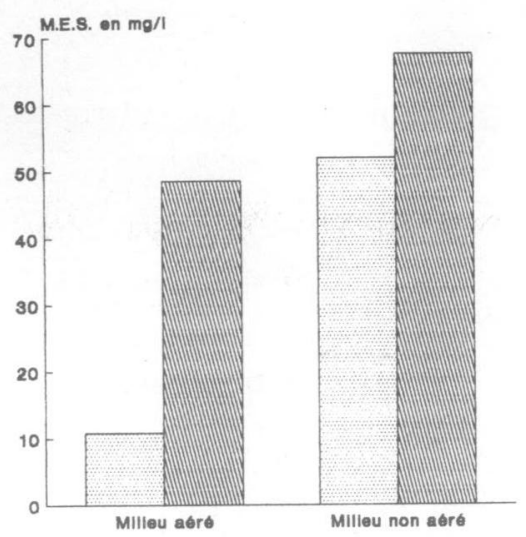

MIIlou aana vera $\quad$ MITHIJ MIlleu avec vers

Fig. 6. Evolution du taux de matières en suspension dans le milieu.

Tableau III. Taux de matières en suspension (en $\mathrm{mg} / \mathrm{l}$ ) dans le milieu, en présence ou non d'Oligochètes et en fonction des conditions d'aération.

\begin{tabular}{lll}
\hline & Milieu aéré $6 \mathrm{j}$ & Milieu non aéré \\
\hline Témoin sans vers & $10,75 \pm 10$ & $51,8 \pm 8$ \\
Milieu avec vers & $48,47 \pm 32,8$ & $67,5 \pm 27,7$ \\
\hline
\end{tabular}

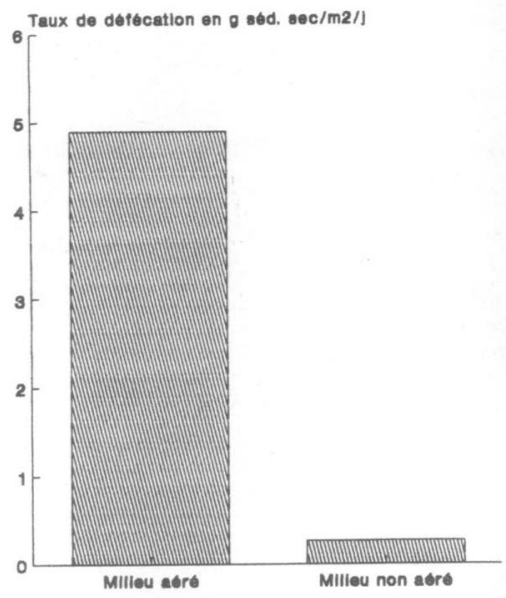

Fig. 7. Influence de l'état d'oxygénation du milieu sur le tauk de défécation des Tubificidae.

Tableau IV. Taux de défécation du sédiment par Tubifex en for tion du temps et des conditions d'aération du milieu.

\begin{tabular}{lcc}
\hline & Milieu aéré $6 \mathrm{j}$. & Milieu non aéré \\
\hline $\begin{array}{l}\text { Taux de défécation } \\
\text { en } \mathrm{mg} \mathrm{sec} / \mathrm{mg} \mathrm{sec} \\
\text { de ver } / \mathrm{j}\end{array}$ & $0,45 \pm 0,2$ & $0,024 \pm 0,009$ \\
$\begin{array}{l}\text { Taux de défécation } \\
\text { en } \mathrm{mg} \mathrm{sec} / \mathrm{m}^{2} / \mathrm{j}\end{array}$ & $4,9 \pm 2,1$ & $0,263 \pm 0,098$ \\
\hline
\end{tabular}

Tableau V. Taux d'excrétion et de défécation (en $\mathrm{mg} / \mathrm{m}^{2} / \mathrm{j}$ ) des différentes formes de phosphore par Tubifex, en fonction des conditions d'aération du milieu.

\begin{tabular}{llrrr}
\hline & Ortho-P & P. orga. diss. & P parti. & P total \\
\hline Milieu aéré & $2,9 \pm 1,2$ & $1,64 \pm 0,76$ & $15,88 \pm 6,99$ & 20,42 \\
Milieu non aéré & $2,9 \pm 0,09$ & $2,72 \pm 1,43$ & $0,59 \pm 0,34$ & 6,21 \\
\hline
\end{tabular}




\subsection{Influence de la désoxygénation du milieu}

\section{Echanges sédiment-eau}

A partir du sixième jour d'expérience, le milieu est appauvri progressivement en oxygène jusqu'au douzième jour, qui marque la fin de l'expérience. Le pourcentage de saturation en oxygène passe ainsi en moyenne de $122 \% \pm 2$ à $45,5 \% \pm 4$ dans les aquariums (Tableau $\mathrm{I}$ ).

La quantité de phosphore total relarguée par le sédiment est significativement plus élevée dans le milieu non aéré que dans le milieu aéré, qu'il y ait ou non des Oligochètes dans le sédiment (fig. 10 , Tableau II). Ce phénomène résulte essentiellement de l'augmentation de la quantité de phosphore particulaire présent dans le milieu. On note d'ailleurs parallèlement que le taux de matières en suspension passe de $1 \mathrm{l}$ à $52 \mathrm{mg} / \mathrm{l}$ dans te milieu sans vers, et de 48,5 à $67,5 \mathrm{mg} / \mathrm{l}$ dans celui qui en contient, lorsque le pourcentage de saturation en oxygène diminue (fig. 6, Tableau III). Par contre, les orthophosphates et le phosphore organique dissous accusent

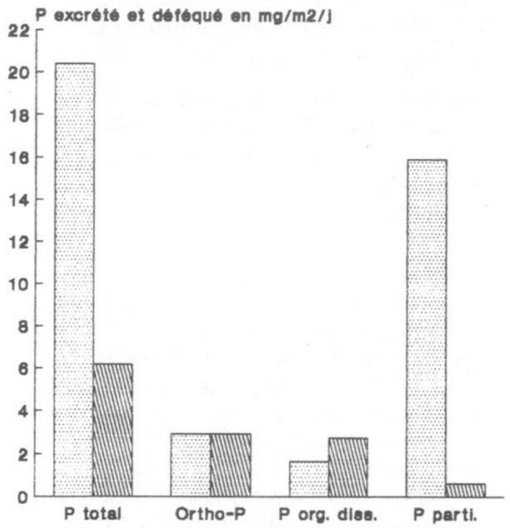

Milieu ate

Milieu non aere

Fig. 8. Intluence de l'état d'oxygénation du milieu sur le taux d'excretion et de défécation des différentes formes de phosphore, par les Tubificidae. une très légère baisse, non significative, au fur et à mesure que l'oxygène dans le milieu, avec ou sans vers, se raréfie (fig. 10).

La proportion des différentes formes de phosphore relarguées par le sédiment évolue considérablement lorsqu'on passe d'un milieu aéré à un milieu qui ne l'est pas (fig. 5). Dans les milieux avec ou sans Oligochètes, le phosphore particulaire passe respectivement de $42 \%$ et $5 \%$ à $80 \%$ et $66 \%$, les orthophosphates de $25 \%$ et $72 \%$ à $11 \%$ et $23 \%$, et enfin le phosphore organique dissous passe de $34 \%$ et $23 \%$ à $9 \%$ et $10 \%$.

Dans le milieu pauvre en oxygène, la quantité de phosphore total libérée par un sédiment qui contient des Tubifex est de $8,92 \mathrm{mg} / \mathrm{m}^{2} / \mathrm{j}$ contre seulement
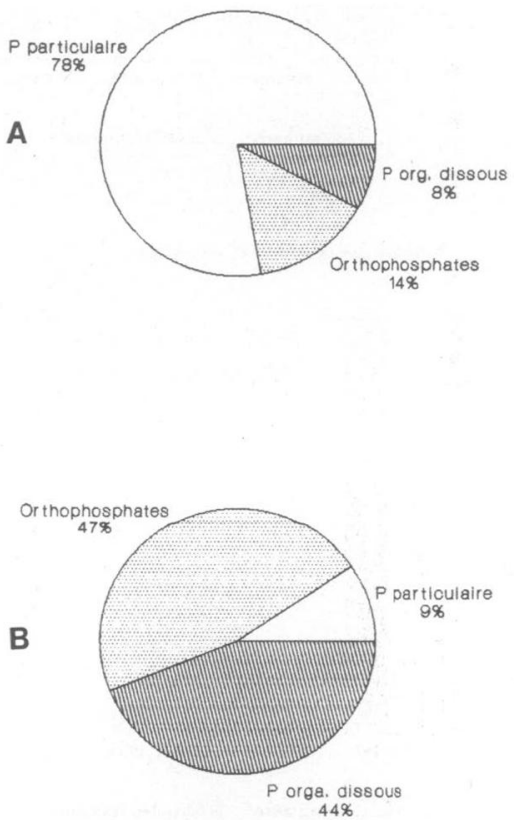

Fig. 9. Répartition des différentes formes de phosphore excrétées et déféquées par les Tubificidae, après leur séjour dans le sédiment. (A : milieu oxygéné ; B : milieu non oxygéné). 


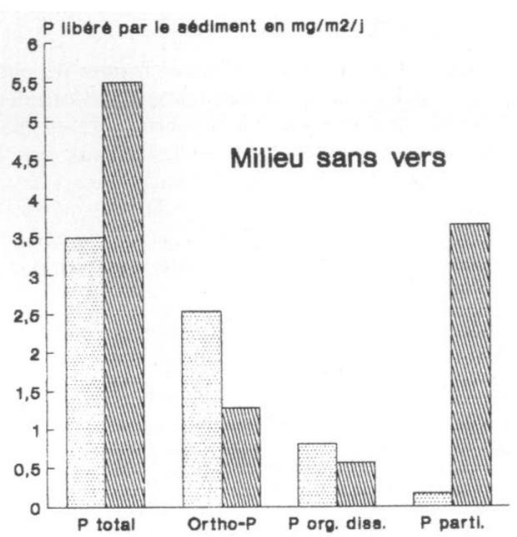

Milieu aeré $\quad$ Millitil Milieu non aeré

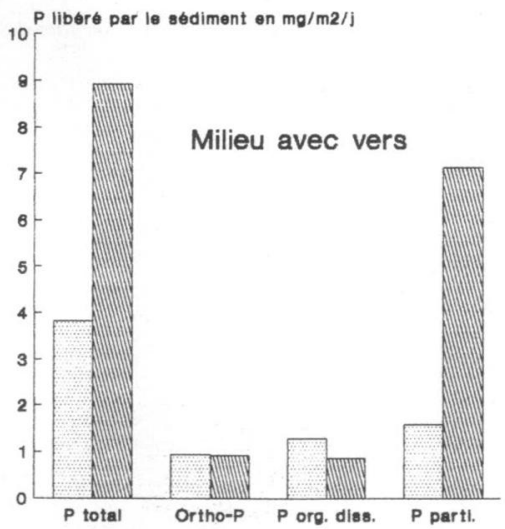

Milieu abre $\quad$ Millill Milieu non abre

Fig. 10. Influence de l'état d'oxygénation du milicu, sur le relargage des différentes formes de phosphore par le sémiment.
$5,48 \mathrm{mg} / \mathrm{m}^{2} / \mathrm{j}$ pour un sédiment qui n'en contient pas (fig. 11, Tableau II B). Les vers sont donc responsables à eux seuls du relargage de $3,44 \mathrm{mg}$ de phosphore par $\mathrm{m}^{2}$ par jour, ce qui représente $38,5 \%$ du phosphore total relargué, contre seulement $8,6 \%$ lorsque le milieu était oxygéné. En fait, le relargage du phosphore, sous forme d'orthophosphates et organique dissous, n'est pas significativement différent entre le milieu contenant des vers et un milieu qui n'en contient pas (fig. 11). Par contre, 7,12 mg de phosphore sont relachés par $\mathrm{m}^{2}$ par jour sous forme particulaire dans un milieu avec Oligochètes, contre seulement $3,63 \mathrm{mg} / \mathrm{m}^{2} / \mathrm{j}$ s'il n'y a aucun vers (fig. 11). Donc $80 \%$ du phosphore relargué par le sédiment, en présence de Tubificidae, est de type particulaire, contre $66 \%$ en absence de vers. Quant aux orthophosphates et au phosphore organique dissous, ils représentent respectivement $11 \%$ et $9 \%$ dans un milieu contenant des vers et $23 \%$ et $10 \%$ dans un milieu n'en contenant pas (fig. $5 \mathrm{~B}$ ).

Finalement, par leur présence, les Oligochètes sont responsables du relargage par le sédiment de $3,49 \mathrm{mg}$ de phosphore sous forme particulaire et de $0,29 \mathrm{mg}$ de phosphore organique dissous, par $\mathrm{m}^{2}$ et par jour, et de la rétention par le sédiment de $0,35 \mathrm{mg}$ d'orthophosphates par $\mathrm{m}^{2}$ par jour (fig. 4).

\section{Excrétion - défécation}

Lorsque le milieu s'appauvrit en oxygène, le taux de défécation diminue significativement et passe de $5 \mathrm{~g}$ de sédiment sec déféqué par $\mathrm{m}^{2}$ et par jour à $0,26 \mathrm{~g} / \mathrm{m}^{2} / \mathrm{j}$ (fig. 7, Tableau IV).

La quaritité de phosphore total libérée directement par excrétion et défécation par les Tubificidae, après 6 jours passés dans un milieu pauvre en oxygène, n'est plus que de $6,21 \mathrm{mg} / \mathrm{m}^{2} / \mathrm{j}$ au lieu des $20,42 \mathrm{mg} / \mathrm{m}^{2} / \mathrm{j}$ qui étaient excrétés et déféqués après un séjour dans de bonnes conditions d'oxygénation (fig. 8, Tableau V). En fait, si la quantité d'orthophosphates excrétée ne change pas, que le milieu soit aéré ou pas, celle de phosphore organique dissous augmente significativement pour atteindre $2,72 \mathrm{mg}$ de phosphore excrété par $\mathrm{m}^{2}$ par jour, alors que celle de phosphore particulaire diminue considérablement : $0,59 \mathrm{mg}$ de phosphore déféqué par $\mathrm{m}^{2}$ par jour au lieu de $15,88 \mathrm{mg}$ lorsque le milieu est bien oxygéné (fig. 8, Tableau V).

Les proportions des différentes formes de phosphore excrétées et déféquées sont donc modifiées lorsque l'on passe d'un milieu bien aéré à un milieu 


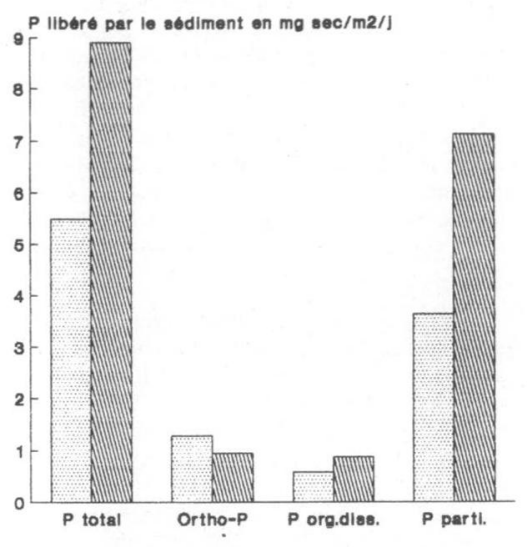

Milieu sans vers $\quad[\mathrm{lll}$ MIII Milieu avec vers

Fig. 11. Influence des Tubificidae sur le relargage des différentes formes de phosphore par le sédiment dans un milieu non oxygéné.

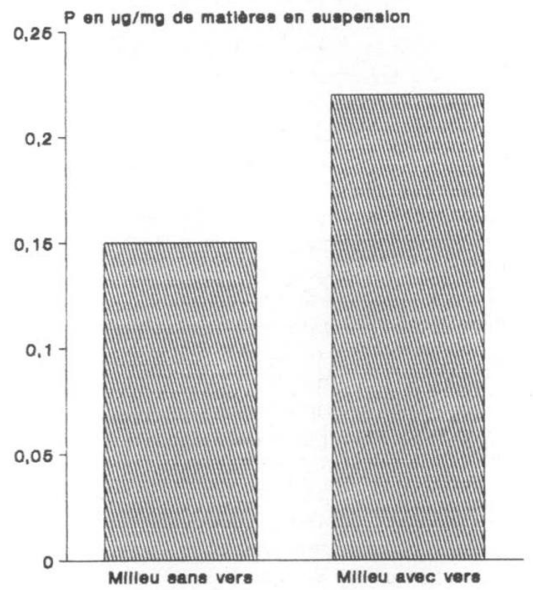

Fig. 12. Phosphore lié aux matières en suspension (en $\mu \mathrm{g} / \mathrm{mg}$ de matières en suspension). pauvre en oxygène (Fig. 9) : sur la totalité du phosphore excrété et déféqué, dans un milieu non aéré, $47 \%$ se trouve sous forme d'orthophosphates, $44 \%$ sous forme organique dissous et $9 \%$ sous forme particulaire, alors que dans un milieu bien aéré, on trouve $14 \%$ du phosphore sous forme d'orthophosphates, $8 \%$ sous forme organique dissous et $78 \%$ sous forme particulaire.

\section{Discussion}

Dans un milieu normalement oxygéné, l'action des oligochètes sur le relargage du phosphore n'apparaît pas significative, au moins sur le plan quantitatif, puisque seulement $8,6 \%$ du phosphore total relargué peut être imputé à la présence des vers. Cette valeur se situe dans la partie inférieure de l'intervalle, très large (8-68\%), des augmentations possibles du taux de phosphore relaché à partir d'un sédiment, dans un lac mésotrophe, grâce à la communauté benthique (Wisniewski \& Planter 1985).

Davis et al. (1975) et Gallepp et al. (1978) ont conclu que les Tubificidae n'intervenaient pas lors du relargage de phosphore par le sédiment. L'étude de l'influence des Chironomidés sur le taux d'échange des phosphates dans un sédiment, confirme que la macrofaune ne semble avoir qu'une importance mineure dans les échanges de nutriments au sein du sédiment (Jacobsen 1977). Cet auteur explique, comme l'a déjà suggéré Kamp-Nielsen (1975), que cet état de fait est le résultat de la combinaison de deux processus directement opposés : le processus de décomposition biochimique et de libération, proportionnel à la densité animale, et le processus d'adsorption qui est fonction de l'augmentation de l'épaisseur de la zone oxydée due aux nombreux tubes de Chironomidés.

Dans notre étude, si l'on prend en compte les différentes formes de phosphore impliquées, les Oligochètes augmentent de façon significative la quantité de phosphore particulaire dans le milieu. Ils induisent parallèlement une rétention significative des orthophosphates par le sédiment : un phénomène annulant l'autre, l'action des vers sur le relargage du phosphore apparaît globalement nulle.

D'un point de vue qualitatif, l'action des Oligochètes reste cependant remarquable. Le phosphore particulaire est huit fois plus important dans le milieu, en présence de vers; inversement, le 
phosphore organique dissous diminue d'un tiers. L'augmentation du phosphore particulaire peut être liée à l'augmentation du taux de matières en suspension observée dans le milieu, lorsqu'il contient des Tubificidae, comme l'avaient déjà noté Holdren \& Armstrong (1980). L'augmentation de la turbidité dans la colonne d'eau, en présence d'organismes benthiques, a d'ailleurs été observée pour la première fois en 1963 par Arrhenius (cité par Krantzberg 1985). Dans un milicu bien aéré, la remise en suspension de matériel sédimentaire, lorsqu'elle est due aux Oligochètes, n'est pas liée aux mouvements qu'effectuent ces derniers, en surface, dans le but de favoriser la circulation de l'eau et donc d'améliorer leur contact avec l'oxygène dissous dans l'eau. L'agitation des vers est alors réduite et ne peut certainement pas avoir d'action significative. En fait, l'activité de fouissage des organismes benthiques, l'ingestion de matériel sédimentaire et la défécation contribuent à augmenter la porosité du sédiment (Krantzberg 1985). Dans la plupart des cas, le sédiment qui a été soumis à la bioturbation contient jusqu'à $90 \%$ d'eau (Rhoads 1974) et il est physiquement instable (Fukuhara 1987) : si le milieu est agité ou subit des courants, il peut être très facilement remis en suspension. Dans nos expériences, le taux de phosphore libéré par la défécation représente $78 \%$ du phosphore émis par les vers (soit $16 \mathrm{mg} / \mathrm{m}^{2} / \mathrm{j}$ ), ce qui explique la hausse du phosphore particulaire dans le milieu en présence de Tubifex.

Les orthophosphates et le phosphore organique dissous représentent respectivement $14 \%$ et $8 \%$ du phosphore total libéré par le métabolisme des vers (soit 2,9 et $1,6 \mathrm{mg} / \mathrm{m}^{2} / \mathrm{j}$ ). Dans des conditions expérimentales similaires, pour une densité de 6685 individus $/ \mathrm{m}^{2}$, soit moitié moins que dans nos expériences, et pour des Chironomidés, Gallepp (1979) a mesuré un taux d'excrétion du phosphore total de $9,4 \mathrm{mg} / \mathrm{m}^{2} / \mathrm{j}$, c'est-à-dire environ la moitié de celui trouvé ici avec Tubifex. Fukuhara \& Yasuda (1985) trouvent, pour Limnodrilus, un taux d'excrétion des orthophosphates de $0,049 \mu \mathrm{g} / \mathrm{mg}$ sec de ver $/ \mathrm{j}$, ce qui équivaut pour la densité de population de nos aquariums à un taux de $0,43 \mathrm{mg} / \mathrm{m}^{2} / \mathrm{j}$, soit six fois moins que le taux mesuré avec Tubifex. Gardner et al. (1981) trouvent un taux d'excrétion du phosphore inorganique, compris entre 0,16 et 0,24 nmole/mg sec de ver/h, et du phosphore organique dissous compris entre 0,07 et $0,12 \mathrm{nmole} / \mathrm{mg}$ sec de ver $/ \mathrm{h}$. Pour une densité de population et un poids sec moyen de Tubifex équivalents aux nôtres, cela correspond à un taux d'excrétion des orthophosphates de 1,3 à $1,96 \mathrm{mg} / \mathrm{m}^{2} / \mathrm{j}$ et du phosphore organique dissous de 0,57 à $0,98 \mathrm{mg} / \mathrm{m}^{2} / \mathrm{j}$. A titre de comparaison, Hylleberg \& Henriksen (1980) ont observé une excrétion des orthophosphates égale à $8{ }_{\mu} \mathrm{mole} / \mathrm{m}^{2} / \mathrm{h}$ soit $5,95 \mathrm{mg} / \mathrm{m}^{2} / \mathrm{j}$, pour le Polychète Nereis virens à une densité de 2000 individus $/ \mathrm{m}^{2}$.

Le phosphore excrété par les organismes benthiques représente 13 à $20 \%$ du phosphore total relargué par le sédiment dans le lac Michigan (Gardner et al. 1981). Dans nos expériences, l'excrétion des orthophosphates et du phosphore organique dissous, due aux Oligochètes, dépasse largement la part du relargage de ces formes de phosphore par le sédiment. Si l'on calcule, pour le phosphore particulaire, organique dissous et pour les orthophosphates, le rapport Excrétion/Relargage défini par Fukuhara \& Sakamoto (1987), on trouve respectivement 9,98, 1,27 et 3,08 . Un rapport supérieur à 1 met en évidence l'importance de l'excrétion dans le processus de relargage du phosphore par le sédiment (Fukuhara \& Sakamoto, op. cit.). Nous avons déjà montré le rôle de la défécation par les Tubificidae sur l'augmentation du phosphore particulaire dans le milieu. Mais la quantité totale de phosphore déféquée reste largement supérieure aux $1,42 \mathrm{mg}$ de phosphore particulaire relargué par le sédiment par $\mathrm{m}^{2}$ et par jour, sous l'action des vers. En fait, comme nous l'avons souligné précédemment, tout ce qui est déféqué ne l'est pas forcément en surface. Ainsi, une partie du phosphore lié aux fécès pourrait rester prisonnière des sédiments alors que l'autre serait remontée en surface, où elle serait disponible, notamment en cas de modifications de l'agitation du milieu. L'excrétion peut également expliquer la part de phosphore organique dissous relarguée par le sédiment et imputée à Tubifex. Mais, là encore, la quantité excrétée est trois fois plus importante que 'a quantité libérée par le sédiment grâce aux Oligochètes. Donc, la plus grande partie du phosphore organique dissous excrétée réellement par les vers, ne se retrouve pas dans la colonne d'eau. Ce phénomène est encore plus marqué pour les orthophosphates. En effet, les Tubificidae provoquent une diminution des orthophosphates dans le milieu, malgré les $2,9 \mathrm{mg} / \mathrm{m}^{2} / \mathrm{j}$ qu'ils excrètent. Cela a été 
observé également par Hylleberg \& Henriksen (1980) avec Nereis virens ainsi que par Davis et al. (1975). Mais pour ces derniers, la disparition de $32 \mathrm{P}$ dans le milieu surnageant, en présence de vers dans le sédiment, n'est peut être que le résultat d'un simple échange isotopique entre le phosphore non marqué, adsorbé à la surface du sédiment, et le $32 \mathrm{P}$ de la colonne d'eau. Le phosphore dissous présente une très grande aptitude à s'adsorber sur les argiles, sur la matière organique inerte, à se fixer sur certains composants du sédiment tels le fer, l'aluminium, le calcium, le manganèse (Holtan et al. 1988, Bonzongo 1989) et à moindre échclle sur la biomasse microbienne (Bonzongo op. cit.). Le phénomène est accéléré lorsque le milieu est agité (Bonzongo op. cit.). Cette capacité d'adsorption du phosphore sur le sédiment et notamment sa réaction de précipitation avec les ions ferriques dans les zones aérobies, permet d'expliquer la diminution des orthophosphates dans l'eau superficielle (Hyllebert \& Henriksen 1980).

En ce qui concerne le phosphore excrété, comme pour la défécation, il est possible également qu'une partie de l'excrétion se fasse à l'intérieur des sédiments, comme cela est suggéré par Wisniewski \& Planter (1985), ce qui expliquerait l'absence presque totale de phosphore dissous excrété par les vers dans le milieu : en présence d'oxygène, il se crée une véritable barrière chimique qui favorise la précipitation d'éléments tels que le phosphore, le fer et le manganèse (Granéli 1979), et limite donc leur relargage dans la colonne d'eau. De plus, la présence d'organismes benthiques permet d'améliorer la diffusion d'oxygène dans les sédiments (Rhoads 1974, Hartgrave 1975, Rippey 1982), ce qui entraîne l'extension en profondeur de la zone oxydée (Edwards 1958, Davis 1974). De cette façon, si le phosphore est excrété à l'intérieur du réseau de galeries des Oligochètes, il peut y rester piégé.

Si une partie du phosphore dissous, excrété dans le sédiment par les vers, est relarguée dans l'eau superficielle ou, si une partie de ce phosphore est directement excrétée en surface oủ l'on ne le retrouve pas, cela signifie qu'il est en totalité adsorbé par le sédiment comme l'observent Anderson et al. (1988) et Fukuhara \& Sakamoto (1987) soit en surface ou au niveau des matières en suspension puisque l'on constate que celles-ci s'enrichissent en phosphore en présence de vers (fig. 12).

Lorsque le milieu s'appauvrit en oxygène, le taux de phosphore particulaire augmente, même si le sédiment ne contient pas d'Oligochètes. Cette augmentation est sans doute liée à l'augmentation de la turbidité dans la colonne d'eau qui traduit une défloculation des sédiments superficiels plus active en milieu réducteur ou une formation de gaz (ébullition) par remontée des zones réductrices méthanogènes par exemple. Ce phénomène sort du cadre classique de la diffusion de phosphore et de fer ferreux en milieu réducteur (Mortimer 1941, 1942) et il se traduit, en fait, par une diminution des orthophosphates disponibles. Il est probablement dûa la nature fortement organique du sédiment. Les autres formes de phosphore ne varient pas significativement.

Par contre, dans le milieu déficient en oxygène, $38,5 \%$ du phosphore total relargué par les sédiments résulte de la présence des vers. Wisniewski \& Planter (1985) donnent une augmentation, entre 34 et $42 \%$, de ce même taux pour un lac eutrophe.

Dans un milieu pauvre en oxygène, l'action des Oligochètes sur le relargage du phosphore est donc significative. Les Tubificidae permettent, comme en milieu bien aéré, une augmentation de la quantité de phosphore particulaire, mais de plus grande amplitude. Or, on constate que le taux de phosphore particulaire déféqué chute considérablement, en même temps que le taux de défécation. L'augmentation du phosphore particulaire dans le milieu vient donc plutôt d'une remise en suspension plus efficace du matériel sédimentaire par les Oligochètes. En effet, lorsque l'oxygène se raréfie dans le milieu, les vers s'extirpent davantage du sédiment et s'agitent très activement pour faciliter les échanges respiratoires (Famme \& Knudsen 1985, Smith 1985 , Anderson et al. 1988). De façon générale, les valeurs d'excrétion du phosphore sont plus hautes en période aérobie lorsque le relargage du phosphore est bas (Wisniewski \& Planter 1985). Cela est vrai dans notre étude en ce qui concerne la défécation puisque le phosphore déféqué est trente fois plus important dans le milieu aéré que dans celui qui ne l'est pas. Par contre, la quantité d'orthophosphates excrétée reste la même, quelles que soient les conditions d'aération. Il est possible qu'elle soit tamponnée par la présence de particules en suspension 
d'où l'absence de différence. Quant au phosphore organique dissous, il est excrété en plus grande quantité par les vers lorsque le milieu s'appauvrit en oxygène.

\section{Conclusion}

Les Tubificidae jouent un rôle considérable dans le déterminisme des formes de phosphore relargué par le sédiment. Grâce à leur activité de fouissage, de défécation ou d'agitation du milieu avec l'extrémité postẹ́rięure de lẹur corps (pour augmenter l'efficacité des échanges respiratoires lorsque le milieu est pauvre en oxygène), les Oligochètes provoquent une augmentation du phosphore particulaire dans la colonne d'eau. Lorsque la teneur en oxygène dissous du milieu est faible, cette augmentation est remarquable puisque $38 \%$ du phosphore total, relargué par le sédiment, résulte de la présence des Tubificidae, et se trouve essentiellement sous forme particulaire. Par contre, si le taux de saturation en oxygène dissous est élevé, l'augmentation du phosphore particulaire, induite par la présence des Oligochètes, est masquée par une diminution de la quantité des orthophosphates qui sont retenus par le sédiment, et fixés sur les particules : de ce fait, l'action des Tubificidae sur le relargage du phosphore par le sédiment semble nulle.

Paradoxalement, c'est lorsque l'influence des Oligochètes est la plus significative, c'est-à-dire lorsque les conditions d'oxygénation sont médiocres, que la quantité de phosphore libéré par excrétion et défécation est la plus faible, alors que dans un milieu bien oxygéné, l'action des vers sur le relargage du phosphore par le sédiment passe inaperçue, bien que la quantité de phosphore libéré par le métabolisme des Oligochètes soit remarquable. Le phosphore relaché de cette façon serait piégé à l'intérieur des sédiments, à leur surface ou au niveau des matières en suspension et resterait non disponible, tant que le milieu ne subit aucune perturbation physique (agitation) ou chimique (baisse de la teneur en oxygène).

Ces résultats posent le problème de la disponibilité du phosphore ainsi relargué pour les algues. $\mathrm{Si}$ seuls les orthophosphates sont biodisponibles, en absence d'agitation du milieu notamment, l'activité des Tubificidae dans les sédiments aurait plutôt un effet négatif. Au contraire, si le phosphore particulaire reste disponible par effet d'échange continu avec la colonne d'eau, l'action des Oligochètes s'avère positive quant à la fourniture de phosphore aux algues.

\section{Remerciements}

Les auteurs remercient M. Narcisse Giani pour ses conseils concernant les Oligochètes, ainsi que Mlle Claude Mur qui a effectué les analyses chimiques.

\section{Travaux cités}

Andersson (G.), Graneli (W.) \& Stenson (J.). 1988. - The influence of animals on phosphore cycling in lake ecosystems. Hydrobiologia, 170: 267-284.

Bonzongo (J.C.J.). 1989. - Phosphore et sédiments : méthodes d'analyse, stockage, relargage, biodisponibilite. Thèse Doct. Univ. Rennes I. 197 p.

Davis (R.B.). 1974. - Tubificids alter profiles of redox potential and $\mathrm{pH}$ in profundal lake sediment. Limnol. Oceamogr., $19: 342-346$.

Davis (R.B.), Thurlow (D.L.) \& Brewster (F.E.). 1975. - Effect of burrowing tubificid worms on the exchange of phosphorus between lake sediment and overlying water. Vehr. Int. Verein. Limnol, $19: 382-394$.

Edwards (R.W.). 1958. - The effects of larvae of Chironomus riparius Meigen on the redox potentials of settled activated sludge. Ann. Appl. Biol., 46: 457-464.

Famme (P.) \& Knudsen (I.). 1985. - Aerotaxis by the freshwater Oligochaete Tubifex sp. Oecologia, 65 : 599-601.

Fukuhara (H.) \& Yasuda (K.). 1985. - Phosphorus excretion by some freshwater zoobenthos dominated in a eutrophic freshwater lake and its dependency, Jpn. J. Limnol., 46 : 287-296.

Fukuhara (H.). 1987. - The effect of Tubificids and Chironomids on particule redistribution of lake sediment. Ecol. Res., 2 : 255-264.

Fukuhara (H.) \& Sakamoto (M.). 1987. - Enhancement of inorganic nitrogen and phosphate release from lake sediment by tubificid worms and chironomid larvae. Oikos, $48: 312-320$.

Gallepp (G.W.), Kitchell (J.F.) \& Bartell (S.M.). 1978. - Phosphorus release from lake sediments as affected by Chironomids. Vehr. Int. Verein. Limnol, 20:458-465.

Gallepp (G.W.). 1979. - Chironomids influence on phosphorus release in sediment water microcosm. Ecology, 60 : 557-566.

Gardner (W.S.), Napela (T.F.), Quiqley (M.A.) \& Malczyk (J.M.). 1981. - Release of phosphorus by certain benthic invertebrates. Can J. Fish. Aqua. Sci., 38 (8): 978-981.

Graneli (W.). 1979. - The influence of Chironomus plumosus larvae on the exchange of dissolved substances between sediment and water, Hydrobiologia, $66: 159-160$.

Hartgrave (B.T.) 1975. - Stability in structure and function of the mud-water interface. Wehr. Int. Verein. Limnol., 19 : 1073-1079.

Holdren (G.C.) \& Armstrong (D.E.). 1980. - Factors affecting phosphorus release from lake sediment cores. Environ. Sci. Technol., $14: 79-87$.

Holtan (H.), Kamp-Nielsen (L.) \& Stuanes (A.O.). 1988. - Phosphorus in soil water and sediment : an overview. Hydrobiologia, $170: 19.34$. 
Hylleberg (J.) \& Henriksen (K.). 1980. - The central role of bioiurbation in sediment mineralization and element recycling. Ophelia, $1: 1-16$.

Jacobsen (O.S.). 1977. - The influence of bottom fauna density on the exchange rates of phosphate and inorganic nitrogen in an eutrophic profundal sediment. Nordiske Sedimenisymposium, Salten Skov, Danmark, 10-13 feb. 1977.

Kamp-Nielsen (L.). 1975. - A kinetic approach to the aerobic sediment-water exchange of phosphorus in lake Esrom. Ecol. Modelling, $1: 153-160$.

Krantzberg (G.). 1985. - The influence of bioturbation on physical chemical and biological parameters in aquatic environments : a review. Environ. Pollut. Ser. A, 39 : 99-122.

McCall (P.L.) \& Fisher (J.B.). 1980. - Effects of Tubificid Oligochaetes on physical and chemical properties of Lake Erie sediment. in Brinkhurst (R.O.) \& Cook (D.G.) eds, Aquatic Oligochaetes biology, Plenum Press, New York : 331-381.

Mortimer (C.H.). 1941. - The exchange of dissolved substances between mud and water in lakes. I. Journal of Ecology, $29: 180-239$.

Mortimer (C.H.). 1942. - The exchange of dissolved substances between mud and water in lakes. II. Journol of Ecology, $30: 147-201$.
Motomizu (S.), Wakimoto (T.) \& Toei (K.). 1983. - Spectrophotometric determination of phosphate in river waters with molybdate and malachite green. Analysi. March. 108 : 361-367.

Peur (T.). 1977. - Bioturbation and exchange of chemicals in the mud water interface. in Golterman (H.L.) ed, Interaction between sediments and freshwater, Junk, The Hague : 216-226.

Rhoads (D.C.). 1974. - Organisms-sediment relation on the muddy sea floor. Oceanogr. Mar. Biol, Ann. Rev., 12 : 263-300.

Rippey (B.) \& Jewson (D.H.). 1982. - The rates of sedimentwater exchange of oxygen and sediment bioturbation in Lough Neagh Northern Ireland. Hydrobiologia, 92 : 377-382.

Smith (M.E.). 1985. - Tubificid worms : important organisms in aquatic ecosystems. The American Biology Teafcher, 47 : 412-415.

Wisniewski (R.J.) \& Planter (M.). 1985. - Exchange of phosphorus across sediment-water interface (with special attention to the influence of biotic factors) in several lakes of different trophic status. Verh. Int. Verein. Limnol, 22 : 3345-3349. 